\title{
Detecção e monitoramento da resistência do tripes Frankliniella occidentalis ao inseticida espinosade
}

\author{
Débora Soller Rais (*); Mário Eidi Sato; Marcos Zatti da Silva \\ Instituto Biológico, Caixa Postal 70, 13001-970 Campinas (SP), Brasil. \\ (*) Autora correspondente: debysrais@gmail.com
}

Recebido: 26/abr./2012; Aceito: 18/fev./2013

\begin{abstract}
Resumo
O tripes Frankliniella occidentalis (Pergande) (Thysanoptera: Thripidae) é uma das pragas mais importantes em cultivos protegidos, principalmente de plantas ornamentais e hortícolas. Entre os problemas enfrentados pelos agricultores estão a dificuldade de seu controle com o uso de inseticidas devido à sua preferência pelas partes internas das flores e a evolução de resistência aos agroquímicos. O objetivo da pesquisa foi caracterizar a resistência de F. occidentalis a espinosade e avaliar a frequência de insetos resistentes ao inseticida, em áreas comerciais de crisântemo de diversos municípios do Estado de São Paulo. Seleções para resistência a espinosade foram realizadas em laboratório, utilizando-se uma população de F. occidentalis, coletada em 2007 de um cultivo comercial de crisântemo em Campinas (SP). No decorrer de sete seleções para resistência, a $\mathrm{CL}_{50}$ de espinosade passou de 8,41 mg i.a. $\mathrm{L}^{-1}$ para $1111 \mathrm{mg}$ i.a. $\mathrm{L}^{-1}$. Comparando-se a linhagem resistente (R) (selecionada) e a suscetível $(S)$, a razão de resistência $\left(C L_{50} R / C L_{50} S\right)$ atingiu valores de $\approx 280$ vezes. Estabeleceu-se uma concentração discriminatória de $98 \mathrm{mg}$ i.a. $\mathrm{L}^{-1}$ para o monitoramento da resistência de F. occidentalis a espinosade. 0 monitoramento foi realizado coletando-se 19 populações do tripes em áreas comerciais de crisântemo no Estado de São Paulo. Os bioensaios foram realizados com ninfas de segundo ínstar de F. occidentalis, colocadas em arenas de folha de feijão. As aplicações de espinosade, na sua concentração discriminatória, foram realizadas sobre as ninfas em torre de Potter. Os resultados indicaram alta variabilidade entre as populações com relação à suscetibilidade a espinosade. Foram observadas populações com até $40,7 \%$ de insetos resistentes. Este é o primeiro relato de resistência de F. occidentalis a espinosade no Brasil.
\end{abstract}

Palavras-chave: monitoramento de resistência, crisântemo, Thripidae.

\section{Detection and monitoring of resistance to the insecticide spinosad in the thrips Frankliniella occidentalis}

\begin{abstract}
The thrips Frankliniella occidentalis (Pergande) (Thysanoptera: Thripidae) is one of the major pests in greenhouses, especially on ornamental plants and vegetables. Among the problems faced by farmers are the difficulties in the control of this pest with the use of insecticides because of its preference for the inner parts of the flowers and the evolution of resistance to agrochemicals. The objective of this research was to characterize the spinosad resistance in F. occidentalis and to assess the frequency of resistant insects in commercial chrysanthemum fields from several counties in the state of São Paulo (SP). Selections for resistance to spinosad were performed under laboratory conditions, using a population of F. occidentalis collected in 2007 in a commercial chrysanthemum field in Campinas (SP). In the course of seven selections for resistance, the LC $_{50}$ of spinosad increased from $8.41 \mathrm{mg}$ a.i. $\mathrm{L}^{-1}$ to $1,111 \mathrm{mg}$ a.i. $\mathrm{L}^{-1}$. Comparing the resistant $(\mathrm{R})$ and the susceptible $(\mathrm{S})$ strains, the resistance ratio $\left(L_{5} R / L C_{50} S\right.$ ) reached values up to 280 -folds. A discriminating concentration of $98 \mathrm{mg}$ a.i. $\mathrm{L}^{-1}$ was established for monitoring the spinosad resistance in F. occidentalis. The survey was conducted by collecting 19 populations of thrips in commercial chrysanthemum fields in the state of São Paulo. The bioassays were conducted with second instar nymphs of F. occidentalis, placed in bean leaf disc arenas. The spinosad was sprayed at the discriminating concentration on the nymphs of thrips, using a Potter spray tower. The results indicated high variability in the susceptibility to spinosad among F. occidentalis populations. Populations with up to $40.7 \%$ of resistant insects were found. This is the first report on spinosad resistance in F. occidentalis in Brazil.
\end{abstract}

Key words: resistance monitoring, chrysanthemum, Thripidae. 


\section{INTRODUÇÃO}

O tripes Frankliniella occidentalis (Pergande) (Thysanoptera: Thripidae) é um inseto de ampla distribuição mundial (CAB INTERNATIONAL, 2010), com registro em mais de 500 espécies de plantas hospedeiras, pertencentes a 50 famílias, incluindo muitas culturas de importância agrícola, como feijão, pimentáo, pepino, berinjela, alface, cebola, tomate, melancia e ornamentais (Yudin et al., 1986; Monteiro et al., 2001; DAisie, 2006; Cab International, 2010). Essa espécie é uma das mais importantes pragas em cultivos protegidos, principalmente de plantas ornamentais (ex. crisântemo) e hortícolas. Altas infestaçôes podem provocar danos consideráveis e grandes perdas de produção, afetando também a qualidade dos produtos e sua comercialização (MurpHy et al., 1998).

Estratégias de controle dessa praga baseiam-se, predominantemente, em aplicaçóes de inseticidas. A utilização intensiva de produtos químicos tem levado a sérios problemas de desenvolvimento de resistência da praga a inseticidas de diferentes grupos químicos, em vários países (Immaraju et al., 1992; Espinosa et al., 2005; Herron e James, 2005; Bielza et al., 2007). No Brasil, relatos de resistência de $F$. occidentalis a inseticidas são raros.

Espinosade é o único inseticida registrado para controle de F. occidentalis em crisântemo no Brasil (Agrofit, 2012). Espinosade possui modo de ação único, sendo ativador alostérico de receptores nicotínicos da acetilcolina. $\mathrm{O}$ inseticida provoca excitaçáo do sistema nervoso dos insetos, levando a contraçôes musculares involuntárias, prostração com tremores, paralisia e finalmente a morte (DOWAGRO, 2010; IrAC, 2012).

Esta pesquisa teve como objetivos caracterizar a resistência de $F$. occidentalis a espinosade e avaliar a frequência de insetos resistentes ao inseticida, em áreas comerciais de crisântemo de diversos municípios do Estado de Sáo Paulo.

\section{MATERIAL E MÉTODOS}

\section{População de tripes e manutenção da criação}

A população utilizada para as seleçôes artificiais foi obtida em área comercial de crisântemo, em Campinas (SP), em setembro de 2007. Após a coleta, os insetos desta espécie foram mantidos e multiplicados em plantas de feijão-de-porco, Canavalia ensiformes L., cultivadas em vasos e mantidas em salas climatizadas $\left(25 \pm 1^{\circ} \mathrm{C}, 70 \pm 5 \%\right.$ de UR e fotofase de 14 horas) em laboratório.

\section{Testes toxicológicos}

Para os bioensaios com $F$. occidentalis, utilizou-se um disco de folha de feijão de $4 \mathrm{~cm}$ de diâmetro, colocado sobre uma camada náo totalmente geleificada de agar-água a $2 \%$, no interior de uma placa de Petri de $6 \mathrm{~cm}$ de diâmetro. Com essa mistura (ágar-água) náo geleificada foi feita uma barreira adicional acompanhando a margem do disco, para impedir a fuga das ninfas. Em cada disco de folha foram colocadas 20 ninfas de segundo ínstar de F. occidentalis. As ninfas (no interior das arenas) receberam a pulverização da calda inseticida (espinosade) $\left(\operatorname{Tracer}^{\mathbb{R}}\right.$ SC 480 g i.a. $\mathrm{L}^{-1}$ ), em torre de Potter (Burkard Manufacturing, Oxford, UK), calibrada à pressão de $68,9 \mathrm{kPa}$. Utilizou-se um volume de $2 \mathrm{~mL}$ da suspensão inseticida, em cada pulverização, obtendo-se uma deposiçáo média de resíduo úmido de $1,6 \mathrm{mg} \mathrm{cm}^{-2} \mathrm{da}$ arena. Após a secagem a $25^{\circ} \mathrm{C}$ à sombra (aproximadamente $15 \mathrm{mi}$ nutos), todas as ninfas foram transferidas com auxílio de pincel de pelo macio para outra arena, não tratada, preparada da mesma maneira como descrita acima, para evitar a fuga das ninfas da arena. As arenas foram mantidas a $25 \pm 2^{\circ} \mathrm{C}, 80 \pm 5 \%$ de UR e fotofase de 14 horas. As avaliações foram realizadas 72 horas após o tratamento.

Foram utilizadas de cinco a sete concentraçóes do inseticida para a obtenção das curvas de concentração-resposta. Os testes foram repetidos pelo menos três vezes. Os resultados foram submetidos à análise de Probit, utilizando-se o programa POLO-PC (LEORA SOFTWARE, 1987).

\section{Seleções para resistência}

A seleção para resistência a espinosade foi feita em laboratório, realizando-se a pulverização do inseticida com torre de Potter, diretamente sobre as ninfas de segundo ínstar de $F$. occidentalis, colocadas em arenas de folha de feijão, conforme descrito anteriormente. Foram colocadas em média 30 ninfas em cada arena tratada.

Foram realizados sete ciclos de seleção artificial, e utilizadas concentraçôes crescentes do inseticida a cada ciclo de seleção, procurando-se obter mortalidades entre 50 e 70\%. Foram utilizados pelo menos 1200 insetos em cada seleção. O intervalo entre uma seleção e outra foi de 30 a 40 dias. Os sobreviventes após 72 horas foram utilizados para a formação de novas colônias. A criação destes insetos foi feita em plantas de feijão, como descrita anteriormente.

Após as sete seleçôes para resistência foi obtida a linhagem resistente (R) de F. occidentalis a espinosade. A linhagem suscetível foi obtida a partir da população de tripes coletada de crisântemo em Campinas e mantida livre da aplicação de inseticidas.

Os dados de mortalidade para as linhagens $\mathrm{S}$ e $\mathrm{R}$ foram submetidos à análise de Probit. A razão de resistência foi obtida através da divisão da $\mathrm{CL}_{50}$ (concentração letal média) da linhagem R pela $\mathrm{CL}_{50}$ da linhagem $S$.

\section{Estimativa de concentração discriminatória}

A concentração discriminatória seria aquela capaz de matar praticamente a totalidade dos indivíduos 
suscetíveis sem afetar os resistentes. Para espinosade, a concentração discriminatória foi estabelecida como aquela correspondente a $\mathrm{CL}_{99}$ do produto, obtida para a linhagem suscetível.

\section{Monitoramento da resistência}

Foram coletadas populaçôes de F. occidentalis em cultivos comerciais de crisântemo, nos municípios paulistas de Araras, Artur Nogueira, Atibaia, Campinas, Cordeirópolis, Holambra e Santo Antônio de Posse, totalizando 19 populaçóes (Tabela 1).

As populações de tripes coletadas em campo foram mantidas em plantas de feijão-de-porco (em vasos), por uma geração antes da realização dos testes toxicológicos. Os testes foram realizados utilizando o método de pulverização direta sobre ninfas de $F$. occidentalis com o auxilio de torre de Potter, conforme descrito anteriormente.

A frequência de resistência foi estimada com base na porcentagem de sobrevivência dos tripes, quando tratados com espinosade na sua concentração discriminatória. Foram utilizados 240 insetos (oito repetiçóes de 30 insetos/arena), para cada população avaliada.

Os dados de percentuais de frequência de resistência $(X)$ obtidos para cada repetiçáo foram transformados em $\operatorname{arc} \operatorname{sen} \sqrt{(X / 100)}$ e submetidos à análise de variância. As médias de frequência de resistência foram comparadas pelo teste de Tukey a $5 \%$ de probabilidade.

Tabela1. Local e datas das coletas de tripes, para o estudo de monitoramento de resistência de $F$. occidentalis a espinosade, em cultivos de crisântemo

\begin{tabular}{|c|c|c|c|}
\hline População (Local) & Código & Data de coleta & Localização \\
\hline Artur Nogueira I & AN I & 25/9/2008 & $22^{\circ} 32^{\prime} \mathrm{S}, 47^{\circ} 09^{\prime} \mathrm{O}$ \\
\hline Artur Nogueira II & AN II & $25 / 9 / 2008$ & $22^{\circ} 32^{\prime} \mathrm{S}, 47^{\circ} 09^{\prime} \mathrm{O}$ \\
\hline Campinas I & Cam I & $25 / 9 / 2008$ & $22^{\circ} 47^{\prime} \mathrm{S}, 47^{\circ} 02^{\prime} \mathrm{O}$ \\
\hline Campinas II & Cam II & 09/10/2009 & $22^{\circ} 47^{\prime} \mathrm{S}, 47^{\circ} 02^{\prime} \mathrm{O}$ \\
\hline Campinas III & Cam III & 13/9/2010 & $22^{\circ} 46^{\prime} \mathrm{S}, 47^{\circ} 03^{\prime} \mathrm{O}$ \\
\hline Campinas IV & Cam IV & $25 / 7 / 2011$ & $22^{\circ} 46^{\prime} \mathrm{S}, 47^{\circ} 03^{\prime} \mathrm{O}$ \\
\hline Atibaia I & Ati I & $26 / 9 / 2008$ & $23^{\circ} 06^{\prime} \mathrm{S}, 46^{\circ} 29^{\prime} \mathrm{O}$ \\
\hline Atibaia II & Ati II & $26 / 9 / 2008$ & $23^{\circ} 06^{\prime} \mathrm{S}, 46^{\circ} 29^{\prime} \mathrm{O}$ \\
\hline Atibaia III & Ati III & 18/09/2009 & $23^{\circ} 06^{\prime} \mathrm{S}, 46^{\circ} 29^{\prime} \mathrm{O}$ \\
\hline Atibaia IV & Ati IV & $06 / 12 / 2011$ & $23^{\circ} 06^{\prime} \mathrm{S}, 46^{\circ} 29^{\prime} \mathrm{O}$ \\
\hline Cordeirópolis I & Cor I & $29 / 5 / 2008$ & $22^{\circ} 28^{\prime} \mathrm{S}, 47^{\circ} 27^{\prime} \mathrm{O}$ \\
\hline Araras I & Ara I & $16 / 9 / 2008$ & $22^{\circ} 21^{\prime} \mathrm{S}, 47^{\circ} 23^{\prime} \mathrm{O}$ \\
\hline Holambra I & $\mathrm{Hol} \mathrm{I}$ & $18 / 9 / 2009$ & $23^{\circ} 35^{\prime} \mathrm{S}, 47^{\circ} 04^{\prime} \mathrm{O}$ \\
\hline Holambra II & Hol II & $18 / 9 / 2009$ & $23^{\circ} 35^{\prime} \mathrm{S}, 47^{\circ} 04^{\prime} \mathrm{O}$ \\
\hline Holambra III & $\mathrm{Hol}$ III & 06/8/2010 & $23^{\circ} 35^{\prime} \mathrm{S}, 47^{\circ} 04^{\prime} \mathrm{C}$ \\
\hline Holambra IV & Hol IV & $24 / 11 / 2011$ & $23^{\circ} 35^{\prime} \mathrm{S}, 47^{\circ} 04^{\prime} \mathrm{C}$ \\
\hline $\begin{array}{l}\text { Santo Antônio de } \\
\text { Posse I }\end{array}$ & SAP I & 18/9/2009 & $22^{\circ} 36^{\prime} \mathrm{S}, 46^{\circ} 55^{\prime} \mathrm{C}$ \\
\hline $\begin{array}{l}\text { Santo Antônio de } \\
\text { Posse II }\end{array}$ & SAP II & $06 / 8 / 2010$ & $22^{\circ} 35^{\prime} \mathrm{S}, 46^{\circ} 55^{\prime} \mathrm{C}$ \\
\hline $\begin{array}{l}\text { Santo Antônio de } \\
\text { Posse III }\end{array}$ & SAP III & 06/8/2010 & $22^{\circ} 35^{\prime} \mathrm{S}, 46^{\circ} 55^{\prime} \mathrm{C}$ \\
\hline
\end{tabular}

\section{RESULTADOS E DISCUSSÃO}

\section{Seleção para resistência}

Antes do início das seleçóes, a concentração letal média $\left(\mathrm{CL}_{50}\right)$ de espinosade para a população de $F$. occidentalis era de 8,41 mg i.a. $\mathrm{L}^{-1}$. Após sete seleçôes para resistência, a $\mathrm{CL}_{50}$ passou para $1111 \mathrm{mg}$ i.a. $\mathrm{L}^{-1}$. Comparando-se a $\mathrm{CL}_{50}$ de espinosade para linhagem resistente, após a seleçáo para resistência, com a $\mathrm{CL}_{50}$ inicial, para a população não selecionada, a razão de resistência foi de 132 vezes (Tabela 2).

Embora não tenha sido realizada nenhuma seleção para suscetibilidade a espinosade, a $\mathrm{CL}_{50}$ da população não selecionada $(S)$ decresceu de $8,42 \mathrm{mg}$ i.a. $\mathrm{L}^{-1}$ para $2,41 \mathrm{mg}$ i.a. $\mathrm{L}^{-1}$, em pouco mais de um ano, indicando que a resistência a espinosade era instável e que na população inicial (coletada de crisântemo) havia uma porcentagem considerável de insetos resistentes. A razão de resistência final, comparando-se a linhagem resistente selecionada e a linhagem suscetível, atingiu 280 vezes.

A $\mathrm{CL}_{90}$ de espinosade, estimada para a linhagem selecionada para resistência, foi de $3.178 \mathrm{mg}$ i.a. $\mathrm{L}^{-1}$ (I.C. a 95\%: 2.601 a $4.082 \mathrm{mg}$ i.a. $\left.\mathrm{L}^{-1}\right)$ equivalente a aproximadamente 33 vezes a concentração recomendada para controle do tripes $F$ occidentalis em crisântemo no Brasil (Agrofit, 2012). Esse resultado indica que, devido à elevada intensidade de resistência obtida com um número pequeno de seleçôes, a resistência de $F$. occidentalis a espinosade pode se tornar um problema sério em um período curto de tempo, em áreas onde são realizadas aplicaçôes frequentes deste produto.

Altas intensidades de resistência de F. occidentalis a espinosade também foram mencionadas por outros autores (Herron e James, 2005; Bielza et al., 2007). Na Espanha, espinosade tem sido amplamente utilizado para o controle de lepidópteros e tripes, após sua introdução em 2002. Devido à alta eficácia para o controle de $F$. occidentalis e os graves problemas de resistência da praga aos outros inseticidas (Espinosa et al., 2005), espinosade tornou-se quase o único inseticida usado contra tripes, para muitos produtores. O uso excessivo do produto, chegando a mais de dez aplicaçóes por safra, levou ao desenvolvimento de populaçôes altamente resistentes em casas de vegetação do sudeste da Espanha, com intensidades de resistência da ordem de milhares de vezes (BiELZA et al., 2007).

\section{Estimativa de concentração discriminatória}

A partir das seleções artificiais foi possível estabelecer uma concentração discriminatória que pode ser adotada em estudos de monitoramento da resistência de F. occidentalis a espinosade no Brasil.

A concentração de $98 \mathrm{mg}$ i.a. $\mathrm{L}^{-1}$, que causou mortalidade de praticamente $100 \%$ dos tripes da linhagem 
Tabela 2. Seleção para resistência a espinosade em F. occidentalis. Número total (n) de insetos (ninfas do 2. ínstar) utilizado para a obtenção das curvas de concentração-resposta; estimativa da $\mathrm{CL}_{50}\left(\mathrm{mg}\right.$ i.a. $\left.\mathrm{L}^{-1}\right)$ e intervalo de confiança (IC) a 95\%; coeficiente angular e erro padrão da média (EP); Qui-quadrado $\left(\chi^{2}\right)$; grau de liberdade (G.L.); fator de resistência (F.R.)

\begin{tabular}{|c|c|c|c|c|c|c|c|}
\hline $\begin{array}{l}\text { Pressão de Seleção } \\
\text { Número }\end{array}$ & $\begin{array}{l}\text { Concentração } \\
\left.\text { (mg i.a. } \mathrm{L}^{-1}\right)\end{array}$ & $\mathbf{n}$ & $\begin{array}{l}\mathrm{CL}_{50} \\
\text { (IC95\%) }\end{array}$ & Coeficiente Angular \pm EP & $\chi^{2}$ & GL & $\mathbf{F R}^{*}$ \\
\hline 0 & - & 420 & $\begin{array}{c}8,41 \\
(6,58-10,7)\end{array}$ & $1,55 \pm 0,07$ & 5,38 & 4 & 1,0 \\
\hline 1 & 12,5 & 480 & $\begin{array}{c}36,1 \\
(26,5-46,7)\end{array}$ & $1,29 \pm 0,05$ & 1,05 & 5 & 4,3 \\
\hline 2 & 49,9 & 420 & $\begin{array}{c}262 \\
(218-310)\end{array}$ & $2,09 \pm 0,08$ & 6,98 & 4 & 31,2 \\
\hline 3 & 144 & 420 & $\begin{array}{c}529 \\
(454-610)\end{array}$ & $2,71 \pm 0,09$ & 7,53 & 4 & 62,9 \\
\hline 4 & 480 & 420 & $\begin{array}{c}616 \\
(525-700)\end{array}$ & $2,88 \pm 0,10$ & 6,11 & 4 & 73,2 \\
\hline 5 & 576 & 420 & $\begin{array}{c}681 \\
(586-784)\end{array}$ & $2,86 \pm 0,12$ & 5,43 & 4 & 81,0 \\
\hline 6 & 672 & 420 & $\begin{array}{c}823 \\
(702-936)\end{array}$ & $2,88 \pm 0,11$ & 0,77 & 4 & 97,9 \\
\hline 7 & 672 & 480 & $\begin{array}{c}1111 \\
(979-1301)\end{array}$ & $2,81 \pm 0,13$ & 3,67 & 4 & 132,0 \\
\hline
\end{tabular}

*Fator de resistência $=\mathrm{CL}_{50}$ após seleçăo dividido pela $\mathrm{CL}_{50}$ inicial (antes da seleçăo)

suscetível e não afetou significativamente os insetos da linhagem resistente (selecionada) de F. occidentalis, foi escolhida para ser utilizada no monitoramento da resistência desta espécie a espinosade (Figura 1).

\section{Monitoramento da resistência}

A avaliação de 19 populaçóes de F. occidentalis, procedentes de cultivos comerciais de crisântemo de diversos municípios do Estado de São Paulo, indicou que a maioria das populações avaliadas ainda estava suscetível ao inseticida espinosade. $\mathrm{Na}$ maioria das populaçóes $(68,4 \%)$ as porcentagens de insetos resistentes foram iguais ou inferiores a $13 \%$; no entanto, apenas $36,8 \%$ das populaçóes não diferiram significativamente $(\mathrm{F}=46,99 ;$ g.l. $=57 ; \mathrm{p}>0,05) \mathrm{da}$ linhagem suscetível (S) de referência (Figura 2).

Cinco populaçóes (ou 26,3\%) coletadas em áreas comerciais de crisântemo tiveram frequências de resistência acima de $20 \%$, e em duas dessas populaçóes, procedentes de Holambra e Santo Antonio de Posse, havia entre 30 e $41 \%$ de tripes resistentes a espinosade (Figura 2).

Foi verificada uma tendência de aumento da frequência de resistência de F occidentalis a espinosade, em crisântemo no Estado de São Paulo nos últimos anos. No caso de Campinas, na população coletada em 2008 havia aproximadamente $0,3 \%$ de tripes resistentes, não diferindo da linhagem suscetível. Nas populaçóes coletadas em 2010 e 2011 as frequências de resistência estavam entre 17 e $25 \%$, diferindo significativamente da população coletada em 2008. Considerando-se as coletas como um todo, verifica-se que as frequências de resistência das populaçóes amostradas em 2008 eram iguais ou inferiores a 3\%, não diferindo da linhagem $S$ (testemunha não selecionada);

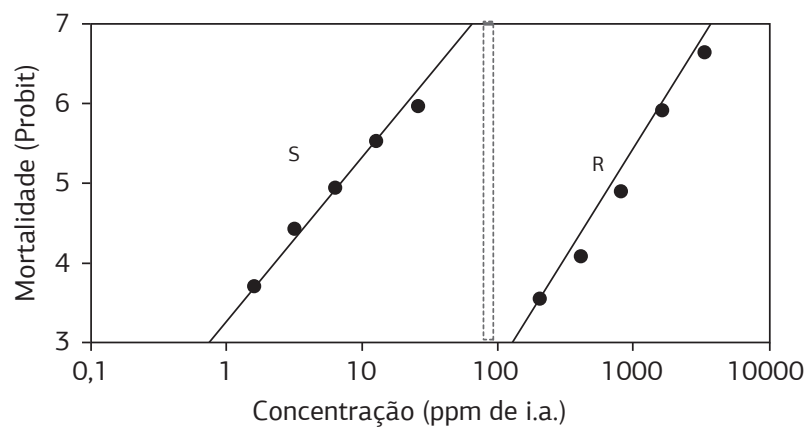

Figura 1. Curvas de concentração-resposta de espinosade em F. occidentalis, para as populaçóes $\mathrm{R}$ (resistente, selecionada com espinosade) e $\mathrm{S}$ (suscetível). As linhas verticais representam uma faixa de concentração discriminatória que pode ser utilizada para o monitoramento da resistência de $F$. occidentalis a espinosade.

em 2010 e 2011, foram observadas populações com até $40 \%$ de insetos resistentes a espinosade. Todas as populaçôes coletadas em 2010 e 2011 diferiram da testemunha.

Loughner et al. (2005) relataram a ocorrência de populaçóes de $F$. occidentalis resistentes a espinosade, em gérbera, em condições de casa-de-vegetação, em diferentes localidades dos Estados Unidos. Algumas populaçóes chegaram a receber oito aplicaçóes de espinosade em um único ano. $\mathrm{Na}$ Espanha, foram encontradas populaçóes de $F$. occidentalis altamente resistentes a espinosade, em casas de vegetação (Bielza et al., 2007). Também foram reportadas populações resistentes de $F$. occidentalis a espinosade, na Austrália (Herron e James, 2005).

Com a confirmação da existência de populaçóes de F. occidentalis resistentes a espinosade em crisântemo no Estado de Sáo Paulo, torna-se relevante a realização de outros estudos como de dinâmica da resistência, para se conhecer como a resistência se comporta em condiçóes de 


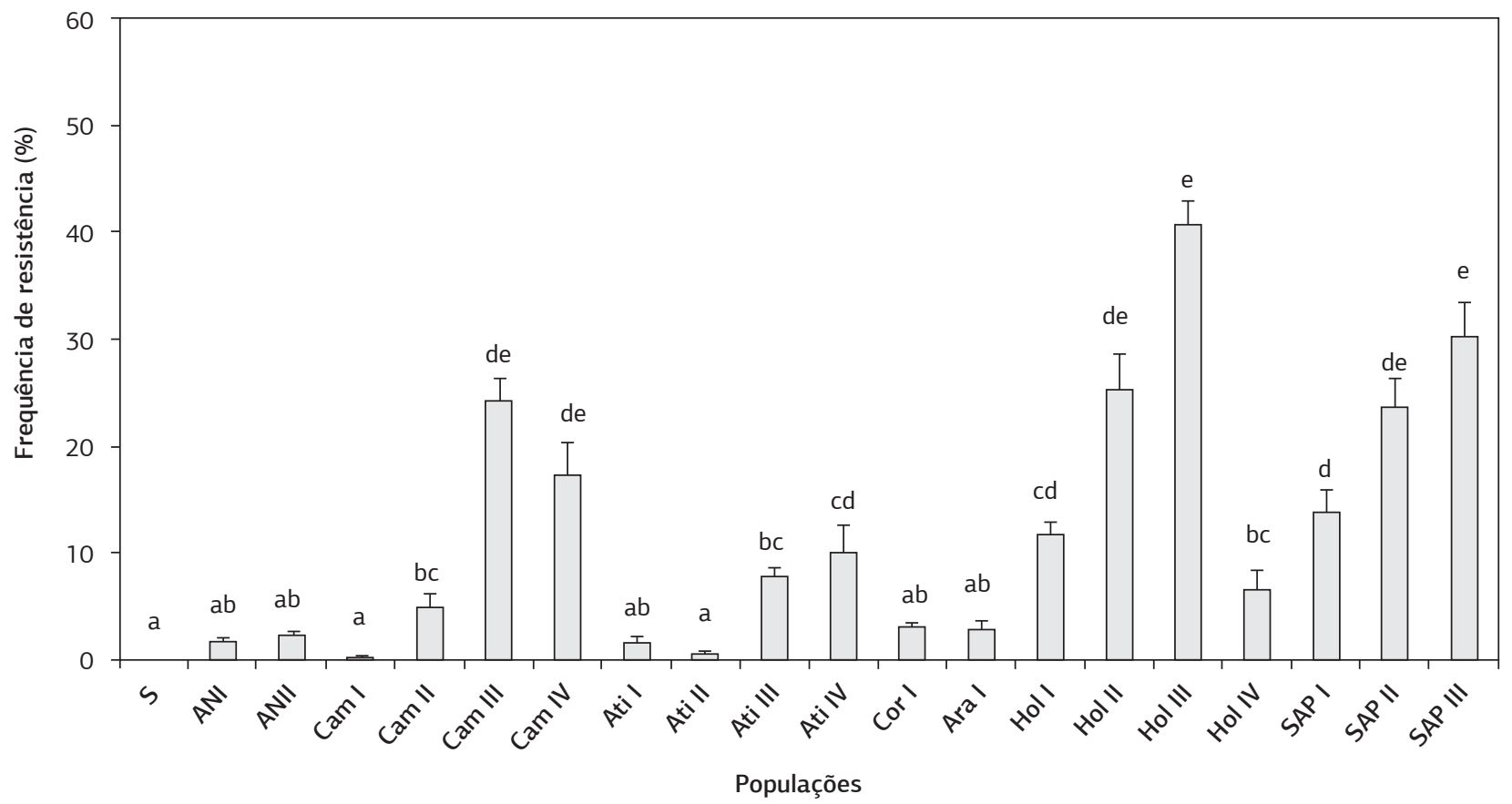

Figura 2. Média de sobrevivência, 72 horas após o tratamento com espinosade na concentração discriminatória de 98 mg i.a. $\mathrm{L}^{-1}$, em várias populaçóes de F. occidentalis coletadas de crisântemo, em diferentes municípios [Artur Nogueira (AN I, AN II), Campinas (Cam I, Cam II, Cam III, Cam IV), Atibaia (Ati I, Ati II, Ati III), Cordeirópolis (Cor I), Araras (Ara I), Holambra (Hol I, Hol II, Hol III, HoIV), Santo Antônio de Posse (SAP I, SAP II, SAP III)], do Estado de São Paulo, no período de 2008 a 2011. População suscetível (S) (Testemunha). Colunas com mesma letra não diferem significativamente entre si a $5 \%$ de probabilidade.

campo. Caso a resistência seja realmente instável, como indicam as avaliaçóes preliminares realizadas com a população original coletada de crisântemo em Campinas (SP), algumas medidas como a rotação de produtos de diferentes mecanismos de ação (IRAC, 2012) poderiam ser exploradas de forma efetiva no manejo da resistência a espinosade.

A concentração discriminatória estabelecida para espinosade pode ser muito útil para o monitoramento da resistência ao inseticida e também para avaliar a eficácia das estratégias de manejo de resistência ao produto a serem adotadas no país.

\section{CONCLUSÃO}

A concentração discriminatória de $98 \mathrm{mg}$ i.a. $\mathrm{L}^{-1}$ pode ser utilizada para o monitoramento da resistência de $F$ occidentalis a espinosade. Em algumas populações de F. occidentalis, coletadas de crisântemo no Estado de São Paulo, havia entre 30 e $40 \%$ de insetos resistentes a espinosade. A maioria das populaçôes é suscetível a espinosade.

\section{AGRADECIMENTOS}

À Fundação de Amparo à Pesquisa do Estado de São Paulo (FAPESP) pelo apoio financeiro à pesquisa (Processos FAPESP 2007/08612-4 e 2012/17972-2) e pelas concessões de bolsas de TT3 e de pós-doutorado ao primeiro e terceiro autores respectivamente. Ao Conselho Nacional de Desenvolvimento Científico e Tecnológico (CNPq) pela concessão da bolsa de produtividade em pesquisa, ao segundo autor.

\section{REFERÊNCIAS}

AGROFIT. Sistema de Agrotóxicos Fitossanitários, do Ministério da Agricultura, Pecuária e Abastecimento. Disponível em: $<$ http:extranet.agricultura.gov.br/ agrofit_cons/principal_agrofit_ cons>. Acesso em: 6/2/2012.

BIELZA, P.; QUINTO, V.; CONTRERAS, J.; TORNE M.; MARTIN, A.; ESPINOSA. P.J. Resitance to spinosad in the western flower thrips, Frankliniella occidentalis (Pergande), in greenhouses of south-aestern Spain. Pest Management Science, v.63, p.682-687, 2007.

CAB International. Distribution Maps of Plant Pests. Disponível em: <http://www.cabi.org/dmpp/default.aspx ?LoadModule = Review \&Review ID = 15424\&site = 164\&pa ge $=1173>$. Acesso em: 26/8/2010.

DAISIE (Delivering Alien Invasive Species Inventories for Europe). Last updated: 2006. Disponível em: <http://www.europe-aliens. org/speciesFactsheet.do?speciesId =51119>. Acesso em: 8/11/2009.

DOWAGRO. Spinosad Overwiew. Disponível em: <http://www. dowagro.com/ turf/prod/spinosad.htm>. Acesso em: 15/6/2010.

ESPINOSA, P.J.; CONTRERAS, J.; QUINTO, V.; GRÁVALOS, C.; FERNÁDEZ, E.; BIELZA, P. Metabolic 
Mechanisms of inseticide resistance in the western flower trips, Frankliniella occidentalis (Pergande). Pest Management Science, v.61, p.1009-1015. 2005.

BIELZA, P.; QUINTO, V.; CONTRERAS, J.; TORNE M.; MARTIN, A.; ESPINOSA. P.J. Resitance to spinosad in the western flower thrips, Frankliniella occidentalis (Pergande), in greenhouses of south-eastern Spain. Pest Management Science, v.63, p.682-687, 2007.

HERRON, G.A.; JAMES, T.M. Monitoring insecticide resistance in Australian Frankliniella occidentalis Pergande (Thysanoptera: Thripidae) detects fipronil and spinosad resistance. Australian Journal of Entomology, v.44, p.299-303, 2005.

IMMARAJU, J.A.; PAINE, T.D.; BETHKE, J.A; ROBB, K.L.; NEWMAN, J.P. Western flower thrips (Thysanoptera: Thripidae) resistance to insecticides in coastal California greenhouses. Journal of Economic Entomology, v.85, p.9-14, 1992.

IRAC (Insecticide Resistance Action Committee) MoA Classification. Scheme. Version 7.2. Issued, April 2012. Disponível em: <http://
www.irac-online.org/wp-content/uploads/MoA-classification.pdf>. Acesso em: 25/4/2012.

LEORA SOFTWARE. Polo-PC: A user's guide to Probit or Logit analysis. Berkeley, 1987. 20p.

LOUGHNER, R.L.; WARNOCK, D.F.; CLOYD, R.A. Resistance of greenhouse, Laboratory, and native populations of Westrtn Flower Thrips to spinosad. HortScience, v.40, p.146-149, 2005.

MONTEIRO, R.C.; MOUND, L.A.; ZUCCHI, R.A. Espécies de Frankliniella (Thysanoptera: Thripidae) de Importância Agrícola no Brasil. Neotropical Entomology, v.1, p.65-72, 2001.

MURPHY, B.C.; MORISAWA, T.A.; NEWMAN, J.P.; TJOSVOLD, S.A.; PARRELLA, M.P. Fungal pathogen controls thrips in greenhouse flowers. California Agriculture, v.52, p.32-36, 1998.

YUDIN, L.S; CHO, J.J.; MITCHELL, W.C. Host range of western flower thrips, Frankliniella occidentalis (Thysanoptera: Thripidae), with special references to Leucaena glauca. Environmental Entomology, v.15, p.1292-1295, 1986. 